\title{
Clinical relevance of thymidylate synthase expression in the signet ring cell histotype component of colorectal carcinoma
}

\author{
D. Cabibi ${ }^{\text {a }}$, A. Calascibetta ${ }^{\text {b }}$, M. Campione ${ }^{\text {a }}$, E. Barresi ${ }^{\text {a }}$, L. Rausa ${ }^{\text {c }}$, G. Dardanoni ${ }^{\text {d, }}$, \\ F. Aragona ${ }^{a}$, R. Sanguedolce ${ }^{b, *}$ \\ a Istituto di Anatomia Patologica, Via del Vespro 129, 90100 Palermo, Italia \\ b Dipartimento di Scienze Farmacologiche, Policlinio Via del Vespro 129, 90100 Palermo, Italia \\ ${ }^{\mathrm{c}}$ Dipartimento di Oncologia Sperimentale e Applicazioni Cliniche, Viale delle Scienze, Facoltà di Medicina e Chirurgia, 90100 Palermo, Italia \\ d Osservatorio Epidemiologico Regionale della Sicilia, Via Vaccaro 5, 90100 Palermo, Italia
}

Received 16 July 2004; accepted 20 July 2004

Available online 7 October 2004

\begin{abstract}
Thymidylate Synthase (TS) is the key enzyme for DNA synthesis pathways and is inhibited by 5-Fluorouracil (5FU). The aim of this work was to study TS expression and the proliferation rate in the different histological types of colorectal carcinoma (CRC). 50 patients with CRC were included in this study and evaluated immunohistochemically using the monoclonal antibodies, TS106 and Ki67. 20 tumours were of the intestinal type, 15 cases were signet ring cell carcinoma (SRCCs) and 15 cases were "mixed-type", with at least two different histological components. Intestinal and mucinous histotypes were positive for TS and Ki67, while "signet ring cell" samples were negative or showed only weak and focal positivity for both the TS and Ki67 antibodies. Our results show that signet ring cells (that are also often present in intestinal and mucinous carcinomas), are in the post-mitotic phase of the cell cycle and show a low proliferation index and TS expression. As TS is the key enzyme for DNA synthesis pathways and is inhibited by 5-fluorouracil (5FU), we can hypothesise that TS expression levels in the different histotypes of CRC could affect the potential responsiveness of these tumours to fluoropyrimidine chemotherapy, with a low efficacy being expected in signet ring cell areas.
\end{abstract}

(C) 2004 Elsevier Ltd. All rights reserved.

Keywords: Thymidylate synthase expression; Signet ring cell carcinoma; Colorectal carcinoma; Immunohistochemistry

\section{Introduction}

Colorectal carcinoma (CRC) is one of the most frequent neoplasms in Western society. The modulation of 5-fluorouracil (5FU) with folinic acid is one of the main therapies for this neoplastic disease, but only 20 $40 \%$ of advanced tumours respond to this treatment [1]. Recently, new strategies have introduced a combination of 5FU with oxaliplatin and/or irinotecan; however, " $5 \mathrm{FU}$ is the most common chemotherapeutic agent used

\footnotetext{
${ }^{*}$ Corresponding author. Tel.: +39 091 6553273; fax +39 091 6553220.

E-mail address: sanguedolce@unipa.it (R. Sanguedolce).
}

in the treatment of colorectal cancer" [2] and today is still present in all drug combinations.

The main target of $5 \mathrm{FU}$ is thymidylate synthase (TS), an enzyme that is necessary for DNA synthesis because it is involved in the pyrimidine synthesis pathway. Nevertheless, the role of TS expression in the response of patients to $5 \mathrm{FU}$ is still debated.

Until now, the literature contains sparse data relating the cancer histotype with the outcome of chemotherapy and the data existing concern only gastric cancer [3]. To the best of our knowledge, TS expression has been related to Dukes' stage in colorectal cancer [4,5], but not to the cancer histotype. Moreover, histological type is not statistically significant in most studies of prognostic 
variables, except for tumour types that are by definition high-grade: specifically, signet ring cell carcinoma (SRCC) and small cell carcinoma [6]. SRCCs are a rare form of CRCs. The incidence rate of the primary SRCC is very low, approximately $2-3 \%$ of all CRCs [7], but the signet ring cell histotype component is frequently present in many intestinal carcinomas.

This component grows in a diffuse fashion, with little or no glandular formation and, in contrast with the pattern in mucinous carcinomas, these tumours synthesise all their mucin in the intracellular space [8]. This intracellular accumulation of mucin results in the displacement of the nucleus and cancer cells acquire the typical signet ring configuration. In some cases, there is a mixture of both extracellular and intracellular mucin formation. Thus, clinical and histological classification of SRCCs remains controversial because they are sometimes considered to be a subtype of "mucinous carcinomas" [9]. More often, they are considered to be a separate histotype, included in the group of CRC rare histotypes with the worse prognoses and with drug-resistant tumours $[6,10]$.

In the present study, we analysed 50 CRCs by means of an immunohistochemistry (IHC) assay to assess TS expression with the aim of identifying a relationship between TS-positivity and the cancer histotype.

As TS is a cell cycle enzyme and is present in proliferating cells [11,12], we also studied the expression of Ki67 (Mib1), a monoclonal antibody which recognises the nuclei of proliferating cells throughout the cell cycle, except during the G0 and early G1 phases, to try to find any relationship between tumour histotype, TS levels, and the proliferation index.

The IHC assay was chosen because it provides the opportunity to study TS and Ki67 expression levels in relation to the morphological features of the areas examined.

\section{Materials and methods}

\subsection{Cases}

The clinical records of 50 patients who underwent surgery for previously untreated colorectal adenocarcinoma, during the time period of January 1999 to December 2003, were reviewed and are summarised in Table 1. No patient had received any prior chemotherapy. Before starting the study, the patient's consent was obtained.

Our study was performed using formalin-fixed, paraffin-embedded primary tumour samples, retrieved from archival material and belonging to our computerised registry of approximately 350 cases of CRC. We included in our observations, all cases previously diagnosed as "signet ring cell colorectal carcinomas" in the abovementioned time period.
Table 1

Clinico-pathological features of patients included in the study

\begin{tabular}{lll}
\hline Patients & & $n$ \\
\hline Gender & M & 31 \\
& F & 19 \\
Age (years) & $<60$ & 12 \\
& $60-70$ & 17 \\
Tumour site & $70>$ & 21 \\
& Right colon & 23 \\
Grading & Left colon & 15 \\
& Rectum & 12 \\
& G1 & 11 \\
Dukes' staging & G2 & 27 \\
& G3 & 12 \\
& A & 5 \\
& B & 16 \\
& C & 24 \\
\hline
\end{tabular}

M, males; F, females.

The histopathological features of the patients' tumours were evaluated by two different pathologists and classified as follows:

Group (A) 20 cases were of the intestinal type, of which 6 were well differentiated, 7 were moderately differentiated and 7 undifferentiated;

Group (B) 15 cases were the signet ring cell type;

Group (C) 15 cases were mixed-type, showing at least two different histological components: 8 cases showed mucinous and intestinal areas, 3 cases showed mucinous, intestinal, and signet ring cell areas and 4 cases showed mucinous and signet ring cell areas.

Each histological component should be present in not less than 10 microscopic high-power fields (HPFs). Each HPF identifies the area of the microscopic field evaluated by a $40 \mathrm{X}$ lens. $\left(1 \mathrm{HPF}=0.159 \mathrm{~mm}^{2}\right)$.

We did not find any pure mucinous tumours because at least $10 \mathrm{HPF}$ showing intestinal and/or signet ring cell components were found in every case with a predominantly mucinous pattern.

\subsection{Immunohistochemistry assay}

For each case, $105-\mu \mathrm{m}$ sections were used from formalin-derived fixed tissue blocks, that were dewaxed and rehydrated. Antigen retrieval was carried out using Dako antigen retrieval fluid and by microwaving at full power for a total of $15 \mathrm{~min}$, divided into 3 periods of $5 \mathrm{~min}$ each. Distilled water was added between each $5 \mathrm{~min}$ of microwaving. Sections were then treated with $3 \%$ hydrogen peroxidase for 5 min, followed by TS 106 (purchased from Neo- 
Markers). This is the antibody that strains for TS and was used at a dilution of $1: 10$ overnight at $4{ }^{\circ} \mathrm{C}$. Visualisation was obtained by incubation with anti-mouse rabbit and goat antibody (Dako) for $15 \mathrm{~min}$, followed by a streptavidin-biotin peroxidase complex (Dako) for $15 \mathrm{~min}$. Finally, Dako chromogen was added for 5 min. Mayer's haematoxylin was used to counterstain the sections. The same TS protocol was used for the antibody Ki67 (purchased from Dako) and used at a dilution 1:50 for $1 \mathrm{~h}$.

As a positive control for both TS and Ki67 staining, we used lymphoid germinal centre cells and normal mucous glands that were present adjacent to tumour. TS staining was evaluated as being positive when the intensity of staining (cytoplasmatic and/or nuclear) was $\geqslant$ the control intensity of staining. Negative controls, stains that lacked primary antibody incubations, were included for every experiment.

TS-positivity for every patient enrolled in groups A and $\mathrm{B}$ was evaluated in 10 microscopic HPFs in which the neoplastic epithelial area should be more than $70 \%$ of the whole field. For each "mixed-type" carcinoma (group C), the evaluation of TS staining was performed separately on 10 HPFs for every histological component.

TS-positivity was evaluated semi-quantitatively as follows:

1. Strongly positive $(+++)$ : if more than $50 \%$ of the neoplastic areas were positive.

2. Mildly positive $(+/++)$ : if $10-50 \%$ of neoplastic areas were positive.

3. Negative $(-/+)$ : if less than $10 \%$ of the neoplastic areas were positive.

In the negative samples (samples with less than 10\% of the neoplastic areas staining positive for TS), single positive cells were counted in $40 \mathrm{HPFs}$ and the mean value in each HPF was reported.

Positivity for Mibl was evaluated according to the percentage of cells showing detectable staining. A semi-quantitative score was adopted:

High proliferation index $(+++)$ : if more than $50 \%$ of nuclei were positive.

Medium proliferation index $(+/++)$ : if $10-50 \%$ of nuclei were positive.

Low proliferation index $(-/+)$ : if less than $10 \%$ of nuclei were positive.

\subsection{Statistical analysis}

Differences in the distribution of the study variables and associations between variables were assessed by means of the $\chi^{2}$ test, with Yates correction when appropriate.

\section{Results}

\subsection{Evaluation of TS and Mib1 expression}

All of the intestinal type carcinomas (group A), independent of the grade of differentiation, showed a widespread positivity in over $50 \%$ of the neoplastic areas evaluated in $10 \mathrm{HPFs}$ and were classified as positive for TS staining $(+++)$. Mib1 was also present in more than $50 \%$ of nuclei, indicating a high proliferation index $(+++)$ (see Fig. 1).

The signet ring cell carcinomas (group B) showed very low TS-positivity, in less than $10 \%$ of neoplastic areas, and very low Mib1-positivity, below 2\% of nuclei staining (range of positivity $0-10 \%$ ). They were classified as negative $(-/+)$ for TS and Mib1 staining (Table 2).

When a count of the absolute number of positive cells was performed in the rare positive areas, the TS staining was very scant and it was mainly nuclear (mean $=4$ cells $\times \mathrm{HPF}$, range $=0-9$ ).

Poor TS and Mib1 expression was more often present in a few undifferentiated cells with large nuclei and little cytoplasm, which were mainly localised in the surface areas of the tumours, whilst cells with a clear "signet ring" morphology were very rarely positive.

Group C showed a different TS and Mib1 expression pattern in different histological components: the intestinal type areas were always positive for TS and Mib1 $(+++)$, the "signet ring cell" areas were almost always negative $(-/+)$; the mucinous areas were heterogeneous for TS expression (ranging from $-/+$ to +++ ) and showed a medium proliferation index $(\mathrm{Mib1}=+/++$ ).

Statistical analysis showed a significant difference between intestinal and signet ring cell cases (groups $\mathrm{A}$ and B) $\left(\chi^{2}=44.4, P<0.0001\right)$.

By combining the immunohistochemical results in the histological areas of the pure carcinomas (groups A and B) with the results of analogous histological areas of mixed carcinomas (group C), a statistically significant difference was obtained between intestinal and signet ring cell areas $\left(\chi^{2}=44.4, P<0.0001\right)$ for both TS and Mib1 expression.

Moreover, a statistically significant association between TS and Mib1 expression in intestinal and signet ring cell areas was found $\left(\chi^{2}=44.4, \quad P<0.0001\right)$ (Table 3).

\section{Discussion}

Until now, the modulation of 5FU with folinic acid has been the main therapy for CRC, but only $20-40 \%$ of advanced tumours will respond to this treatment [1]. Moreover, despite adjuvant 5FU therapy, approximately $30 \%$ of patients with International Union against Cancer (UICC) stage II and 
(a)

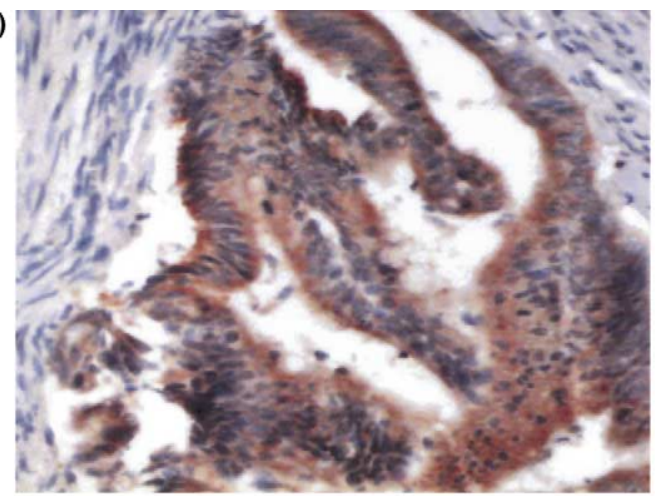

(c)

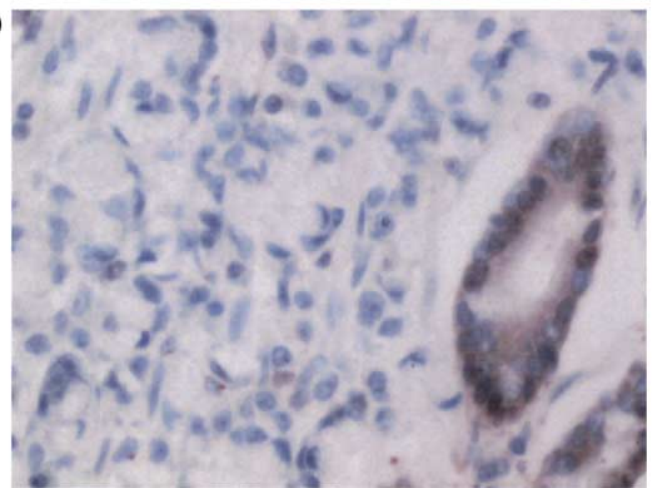

(b)

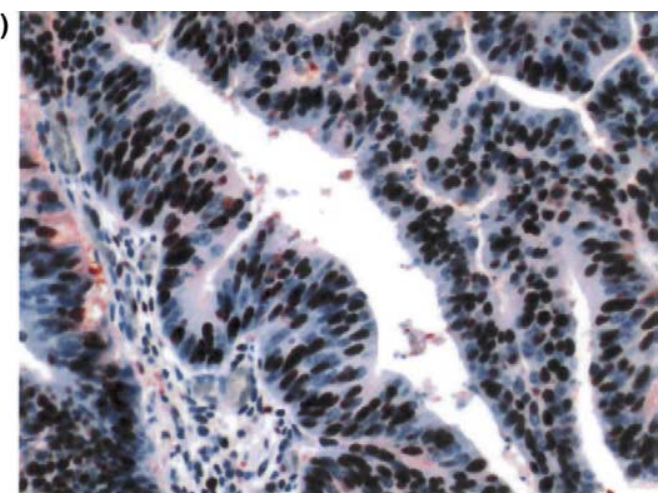

(d)

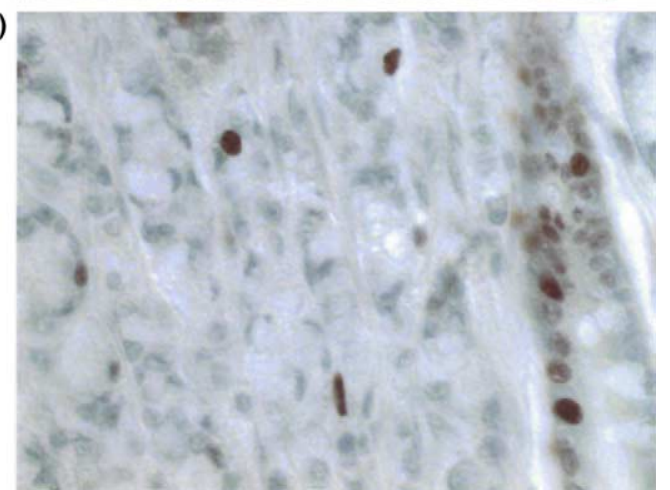

Fig. 1. (a, b) Intestinal type CRC: strong TS staining (a) and Mib1 staining (b) Original magnification: 200× (c, d) Signet ring cell type CRC; negativity for TS (c) and Mib1 (d) in the centre and the left-hand side of the frames. Original magnification: 400×. Observe positive non-neoplastic glands on the right-hand side of the frames (positive controls).

III colorectal cancers recur [13]. New drug combinations of $5 \mathrm{FU}$ combined with oxaliplatin and/or irinotecan have been introduced, but $5 \mathrm{FU}$ is, to date, the most common chemotherapeutic agent used in treatment protocols.

The different response rates to $5 \mathrm{FU}$ have resulted in the need for prognostic markers to define those patients most likely to benefit from chemotherapy. Particular care has been focused on the expression of TS, a crucial target for 5FU in the pathway of pyrimidine synthesis, which is necessary for DNA synthesis. TS expression has been associated with clinical outcome in local and disseminated colorectal cancers.

Previous studies have shown that patients with advanced and/or metastatic disease and high TS contents (probably associated with TS gene amplification) do

Table 2

TS- and Mib1-positivity in groups A and B

\begin{tabular}{lll}
\hline Histotype & TS & Mib1 \\
\hline Group A: 20 cases (intestinal) & +++ & +++ \\
Group B: 15 cases (signet ring cell) & $-/+$ & $-/+$ \\
\hline
\end{tabular}

$(+++)=$ positivity $>50 \%$ of neoplastic areas for $\mathrm{TS}$; positivity $>50 \%$ of nuclei for Mib1(Ki67)

$(-/+)=$ positivity $<10 \%$ of neoplastic areas for TS; positivity $=10 \%$ of nuclei for Mib1 (Ki67)

(Statistically significant difference between intestinal type and signet ring cell carcinomas: $\chi^{2}=44.4, P<0.0001$ ). not respond to 5FU [13-19]. However, many patients with low TS expression also fail to respond to treatment [20].

Other adjuvant treatment studies have stated that patients with high TS levels may benefit from adjuvant 5FU-based chemotherapy [21-23], whereas patients with low TS levels seem to have a worse outcome when treated with such adjuvant chemotherapy [22]. These data agree with recent adjuvant therapy studies in pancreatic [24] and breast cancers [25].

Our study showed that all CRCs of intestinal types (irrespective of the grade of differentiation or intestinal location) and approximately half of the mucinous carcinomas were positive for TS.

By contrast, all SRCCs (irrespective of the intestinal location) were negative for TS (weak cytoplasmic or nu-

Table 3

Group C: TS and Mib1 expression in the mixed-type carcinomas

\begin{tabular}{lll}
\hline & TS & Mibl \\
\hline Intestinal type areas & +++ & +++ \\
Signet ring cell & $-/+$ & $-/+$ \\
Mucinous & $-/+,+/++,+++$ & $+/++$ \\
\hline
\end{tabular}

$(+++)=$ positivity $>50 \%$ of neoplastic areas for $\mathrm{TS},>50 \%$ of nuclei for Mib1

$(+/++)=$ positivity in $10-50 \%$ of neoplastic areas for TS, in $10-50 \%$ of nuclei for Mib1

$(-/+)=$ positivity $<10 \%$ of neoplastic areas for TS, $<10 \%$ of nuclei for Mib1. 
clear stain was present only focally, or in superficial areas or, sometimes, confined to small tumour cells with dark-staining cytoplasm, but "typical" signet ring cells were negative).

TS is a cell cycle enzyme and is present in proliferating cells $[11,12]$. Previous experimental observations in cell lines showed that "the expression and activity of the TS protein in asynchronously-growing cancer cells are significantly related to the cell doubling time; the faster the cell proliferation, the greater the expression and activity of TS" [11].

Our data showed the same relationship between cell proliferation, (showed by Mib1) and TS-positivity. In particular, high positivity for both antibodies was found in intestinal areas; variable TS-positivity and intermediate Mib1-positivity in mucinous areas, and very low positivity in signet ring cell areas.

The low TS- and Mibl-positivity in signet ring cell areas, suggesting a low proliferative activity, was unexpected due to the well known aggressiveness of SRCCs. However, our findings agree with a previous autoradiographic study studying the labelling index in gastric cancers; typical signet-ring cells had a zero labelling index, suggesting that they were non-cycling cells and were functionally differentiated [26].

According to these observations, it can be hypothesised that SRCCs, consist mainly of non-replicating cells that are in a post-mitotic phase.

Thus, mechanisms other than proliferation, (for example, a decrease in apoptosis) could be responsible for the poor prognosis of SRCC, and this needs to be investigated further.

Our data agree with other experimental studies in nude mice subcutaneously (s.c.) injected with human colon cancer HT29 cells, showing that 5FU induced an accumulation of signet ring cells and a depletion of the enterocyte stem cell phenotype only. Since very few signet ring cells were Ki67-positive, the authors hypothesised that "The majority of these cells were non-replicating and thus less sensitive to 5FU treatments" [27].

Further, recent studies assessed that cell lines with faster doubling times had higher TS levels and were significantly more sensitive to 5FU [28]. On this basis, we can hypothesise that since non-replicating signet ring cells have a low TS content, they could be less sensitive to $5 \mathrm{FU}$ because the target of one of the most important modes of action of 5FU (that is the ability of the drug to inhibit DNA synthesis by binding and inactivating the TS enzyme) is lacking in these cells.

Of course, we underline the necessity to develop a better understanding of the other possible mechanisms of the 5FU action in signet ring cells; for example, an increase in the apoptotic pathway. Perhaps in non-proliferating SRCC, this mechanism could be more important than the inhibition of DNA synthesis.
Although data in the literature state that the incidence of primitive SRCC is very low, approximately, $3 \%$ of all CRCs [7], a SRC component is often associated with the intestinal and mucinous histotypes in mixed carcinomas. We think that TS expression levels in the different histotypes can affect the patient's response to 5FU, with a likely low efficacy in signet ring cell areas. If our conclusions are correct, we think it will be important to note any areas of SRCC present in the carcinomas. However, clinical studies are necessary to clarify the real meaning of the presence of the signet ring cell histotype in the patient's response to $5 \mathrm{FU}$.

\section{Conflict of Interest Statement}

None declared.

\section{Acknowledgements}

This work was partially supported by an ex $60 \%$ grant, in 2002, Università di Palermo and by the fellowship entitled "Marilu' Parlavecchio Comito".

\section{References}

1. Moertel CG. Chemotherapy for colorectal cancer. New England $J$ Med 1994, 330, 1136-1143.

2. Mariadason JM, Arango D, Shi Q, Wilson AJ, Corner GA, Nicholas C, et al. Gene expression profiling-based prediction of response of colon carcinoma cells to 5-fluorouracil and camptothecin. Cancer Res 2003, 63(24), 8791-8812.

3. Yagawa A. Histopathological studies of antitumour effect of Tegafur administered by intravenous infusion. Nippon Gan Chiryo Gakkai Shi 1990, 25, 1197-1203.

4. Edler D, Blomgren H, Allegra CJ, Johnston PG, Lagersted T, Magnusson I, et al. Immunohistochemically determination of thymidylate synthase in colorectal cancer, methodological studies. Eur J Cancer 1997, 33, 2278-2281.

5. Araki Y, Isomoto H, Shirouzu K. Dihydropyrimidine dehydrogenase activity and thymidylate synthase levels associated with response to 5-fluorouracil in human colorectal cancer. Kurume Med $J$ 2001, 48, 93-98.

6. Compton CC, Fielding LP, Bugart L, Conley B, Cooper HS, Hamilton SR, et al. Prognostic factor in colorectal cancer. College of American Pathologists Consensus Statement 1999. Arch Pathol Lab Med 2000, 124, 979-994.

7. Nada Sharma NHH et al. Primary signet ring cell carcinoma of the colon and rectum. Histopathology 1995, 26, 378-379.

8. Laufman $\mathrm{H}$ et al. Primary linitis plastic type of carcinoma of the colon. Arch Surg 1951, 62, 79-91.

9. Stemberg SS. Diagnostic Surgical Pathology. Philadelphia, Lippincot William and Wilkins, 1999.

10. Rosai J. Hackerman's Surgical Pathology. Mosby Year Book 1996, 1.

11. Pestalozzi BC, Mc Ginn CJ, Kinsella TJ, Drake JC, Glennon MC, Allegra $\mathrm{CJ}$, et al. Increased thymidylate synthase protein levels are principally associated with proliferation but not cell cycle 
phase in asynchronous human cancer cells. Br J Cancer 1995, 71(6), 1151-1157.

12. Mc Ginn CJ, Pestalozzi BC, Drake JC, Glennon MC, Kunugi K, Otterson $\mathrm{G}$, et al. Cell cycle regulation of the G0/G1 transition in 5-fluorouracil-sensitive and resistant human colon cancer cell lines. Cancer $J$ 2000, 6, 234-242.

13. Johnston PG, Lenz HJ, Leichman CG, Danenberg KD, Allegra JC, Danenberg JC, et al. Thymidylate synthase gene and protein expression correlate and are associated with response to 5fluorouracil in human colorectal and gastric cancer. Cancer Res 1995, 55, 1407-1421.

14. Aschele C, Lonardi S, Monfardini S. Thymidylate synthase expression as predictor of clinical response to fluoropyrimidinesbased chemotherapy in advanced colorectal cancer. Cancer Treat Rev 2002, 28, 27-47.

15. Peters GJ, van der Wilt CL, van Triest B, Codacci Pisanell G, Iohnston PG, van Croeningen $\mathrm{CJ}$, et al. Thymidylate synthase and drug resistance. Eur J Cancer 1995, 31A, 1229-1305.

16. Paradiso A, Simone G, Petroni S, Leone B, Vallejo C, Lacava J, et al. Thymidylate synthase and $\mathrm{p} 53$ primary tumour expression as predictive factors for advanced colorectal cancer patients. $\mathrm{Br}$ Cancer 2000, 82, 560-567.

17. Edler D, Kressner U, Ragnhammar P, Johnston PG, Magnusson I, Glimelius I, et al. Immunohistochemically detected thymidylate synthase in colorectal cancer: an independent prognostic factor of survival. Clin Cancer Res 2000, 6, 488-492.

18. Edler D, Hallstrom M, Johnston PG, Magnusson I, Ragnhammar $\mathrm{P}$, Blomgren P. Thymidylate synthase expression: an independent prognostic factor for recurrence, distant metastasis, disease-free and overall survival in rectal cancer. Clin Cancer Res 2000, 6(4), $1378-1384$.

19. Lenz HJ, Danenberg KD, Leichmann CG, Florentine B, Johnston $\mathrm{S}$, Groshen S, et al. P53 and thymidylate synthase expression in untreated stage II colon cancer association with recurrence, survival and site. Clin Cancer Res 1998, 4(5), 1227-1234.

20. Backus HH, van Riel JM, van Groeningen CJ, Vos W, Dukers $\mathrm{DF}$, Bloemena DF, et al. Rb, mcl-1 and p53 expression correlate with clinical outcome in patients with liver metastases from colorectal cancer. Ann Oncol 2001, 12(6), 779-785.

21. Johnston PG, Fisher ER, Rockette HE, Fisher B, Wolmark JC, Drake JC, et al. The role of thymidylate synthase expression in prognosis and outcome of adjuvant chemotherapy in patients with rectal cancer. J Clin Oncol 1994, 12(12), 2640-2647.

22. Edler D, Glimelius B, Hallstrom M, Jacobsen A, Johnston PG, Magnusson I, et al. Thymidylate synthase expression in colorectal cancer: a prognostic and predictive marker of benefit from adjuvant fluorouracil based chemotherapy. J Clin Oncol 2002, 20, 1721-1728.

23. Kornmann M, Schwabe W, Sander S, Kron M, Strater J, Polat S, et al. Thymidylate synthase and dihydropyrimidine dehydrogenase mRNA expression levels: predictors for survival in colorectal cancer patients receiving adjuvant 5-fluorouracil. Clin Cancer Res 2003, 9(11), 4116-4124.

24. Hu YC, Komorowski RA, Graewin S, Hostetter G, Kallioniemi OP, Pitt OP, et al. Thymidylate synthase expression predicts the response to 5-fluorouracil-based adjuvant therapy in pancreatic cancer. Clin Cancer Res 2003, 9(11), 4165-4171.

25. Foekens JA, Romain S, Look MP, Martin PM, Klijn JG. Thymidine kinase and thymidylate synthase in advanced breast cancer: response to tamoxifen and chemotherapy. Cancer Res 2001, 61, 1421-1425.

26. Sasaki K. An autoradiographic study on the labelling index of biopsy specimens from gastric cancer. Cancer 1994, 54(7), 1307-1309.

27. Sharma R, Adam E, Shumacher U. The action of 5-fluorouracil on human HT 29 colon cancer cells grown in SCID mice: mitosis, apoptosis, and cell differentiation. Br J Cancer 1997, 76(8), 1011-1016.

28. Grem JL, Danenberg KD, Behan K, Parr A, Young L, Danenberg $\mathrm{PV}$, et al. Thymidine kinase, thymidylate synthase, and dihydropyrimidine deydrogenase profiles of cell lines of the National Cancer Institute's Anticancer drug screen. Clin Cancer Res 2001, 7, 999-1009. 